\title{
SUBJECTIVE APPROACH TO OPTIMAL CROSS-SECTIONAL DESIGN OF BIODEGRADABLE MAGNESIUM ALLOY STENT UNDERGOING HETEROGENEOUS CORROSION
}

\author{
Najmeh Zarei ${ }^{a}$, Seyed Ahmad Anvar ${ }^{a, *}$, Sevan Goenezen $^{b}$ \\ a Shiraz University, Department of Civil and Environmental Engineering, Shiraz 71348, Iran \\ ${ }^{b}$ Texas A \& $M$ University, Department of Mechanical Engineering, College Station, 77843 Texas, USA \\ * corresponding author: anvar@shirazu.ac.ir
}

\begin{abstract}
Existing biodegradable Magnesium Alloy Stents (MAS) have several drawbacks, such as high restenosis, hasty degradation, and bulky cross-section, that limit their widespread application in a current clinical practice. To find the optimum stent with the smallest possible cross-section and adequate scaffolding ability, a 3D finite element model of 25 MAS stents of different cross-sectional dimensions were analysed while localized corrosion was underway. For the stent geometric design, a generic sine-wave ring of biodegradable magnesium alloy (AZ31) was selected. Previous studies have shown that the long-term performance of MAS was characterized by two key features: Stent Recoil Percent (SRP) and Stent Radial Stiffness (SRS). In this research, the variation with time of these two features during the corrosion phase was monitored for the 25 stents. To find the optimum profile design of the stent subjectively (without using optimization codes and with much less computational costs), radial recoil was limited to $27 \%$ (corresponding to about $10 \%$ probability of in-stent diameter stenosis after an almost complete degradation) and the stent with the highest radial stiffness was selected.

The comparison of the recoil performance of 25 stents during the heterogeneous corrosion phase showed that four stents would satisfy the recoil criterion and among these four, the one having a width of $0.161 \mathrm{~mm}$ and a thickness of $0.110 \mathrm{~mm}$, showed a $24 \%-49 \%$ higher radial stiffness at the end of the corrosion phase. Accordingly, this stent, which also showed a $23.28 \%$ mass loss, was selected as the optimum choice and it has a thinner cross-sectional profile than commercially available MAS, which leads to a greater deliverability and lower rates of restenosis.
\end{abstract}

KEYWORDS: Coronary stent, biodegradation, magnesium, pitting corrosion, finite element.

\section{INTRODUCTION}

Recently, using magnesium alloy stents in clinical treatments has become relatively common owing to their biosafety, biocompatibility, superior mechanical properties, and comparatively larger stiffness than other metallic and polymeric biodegradable stents. Although Mg alloy stents have shown promising results in stent implantation in clinical trials, relatively high rates of vessel lumen loss, a premature loss of scaffolding ability, and especially bulky cross-sectional profiles remain to be major shortcomings of MAS [1 3].

It is noteworthy that either of the two or both the following approaches can be adopted to improve mechanical and clinical performances of MAS; (1) the development of new materials to enhance the mechanical strength and alter the stent degradation rate, and (2) optimizing the shape of the stent and it's cross-sectional geometry to reduce the rate of lumen loss and the bulkiness of the MAS and also to increase their scaffolding ability [4, 5]. In line with the second approach, the role of the device design as a key indicator for determining the long term scaffolding behaviour has to be studied further.

In recent years, only a few studies have focused on the influence of the stent design and geometry on the mechanical performance of MAS. In the study conducted by Gastaldi et al. [6], a numerical simulation of the behaviour of MAS was investigated based on uniform corrosion and phenomenological-stress corrosion mechanism. The results obtained from these numerical simulations were validated against the experimental corrosion test [7]. Furthermore, Wu et al. 8] applied a combined 3D FEM with a degradable material model to three different MAS designs. They compared the mechanical performance of the three designs while corrosion was in progress. In another study, Grogan et al. 9] developed a numerical model for predicting the effects of a pitting corrosion mechanism on the mechanical performance of MAS.

$\mathrm{Wu}$ et al. 10 proposed a shape optimization method for two-dimensional (2D) finite element model of a single stent hinge without considering corrosion. It should be emphasized that they used a morphing procedure to simplify the optimization and compared the results for four different Mg alloys: AZ31, AZ80, WE43, and ZM21. In another research, Grogan et al. [11] developed a new method to simulate uniform corrosion by employing an Arbitrary LagrangianEulerian (ALE) adaptive meshing technique. The Abaqus/Standard implicit solver was used in their study. In addition, they combined the uniform corro- 


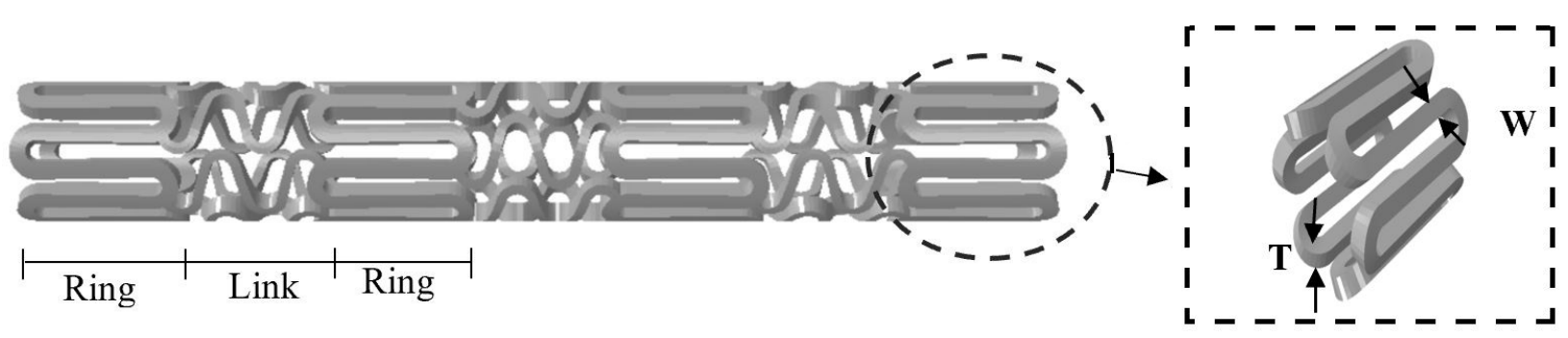

(A).

(B).

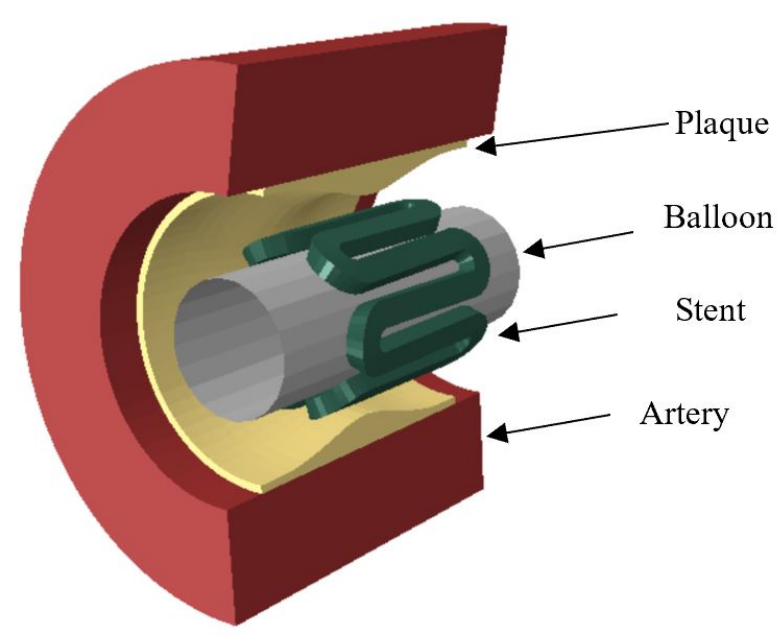

(c).

Figure 1. Stent geometry and numerical model: (A) Complete unit; (B) Single ring used in 3D model; (C) FE 3D model of the investigated system.

sion model based on ALE adaptive meshing with an optimization strategy to find out the relationship between the geometry and the mechanical performance of MAS.

Chen et al. 12 developed a 2D shape optimization framework for MAS with a generic sine-wave design without considering corrosion in the optimization process. They concluded that the complete deformation history, the deformation induced by deployment and crimping, had a significant effect on the mechanical performance of MAS and should be considered in its simulation.

Recently, Ni et al. [13] investigated the effects of the degradation of the stent on the biomechanical performance in the stent-bile duct coupling system. They developed a numerical model based on a continuum damage approach for a biodegradable magnesium alloy bile duct stent. Furthermore, they showed how the computational methods can be used as a powerful tool for studying the degradation mechanism of a magnesium alloy stent in the nonvascular cavities.

By looking at the influence of the stent design and geometry, it may be reasonable to identify the stimulus for the poor efficacy of MAS due to corrosion and to determine suitable biomechanical baseline designs for absorbable magnesium stents. In the present study, 3D finite element models of a biodegradable magnesium alloy stent were developed, which explicitly accounted for localized pitting corrosion and were deployed in idealized stenosed vessels. In current fi- nite element simulations, a continuum damage-based corrosion model was applied, which can fully capture the localized pitting attack [9]. The overall goal of this research is to subjectively determine the most appropriate stent profile concerning its radial recoil, stiffness properties, and mass loss of the stent when localized or pitting corrosion is in progress. The stent profile selection was based on the influence of the stent strut profile on the long-term properties of MAS due to localized corrosion, which is investigated through a parametric study. This parametric study focuses on a ring of the stent with different randomly selected strut widths and thicknesses subjected to pitting corrosion and determining the impairment of the stent recoil and radial stiffness due to pitting corrosion.

\section{Methods}

\subsection{Stent's GEOMETRY And Finite ELEMENT MODEL}

Generally, the stent is made up of two components; the tubular-like rings and the connecting links, Fig. 1a. As stated by Petrini et al. [14, the tubular-like ring scaffolds the vessel after the expansion, whereas the connecting links provide the flexibility of the stent during the delivery of the stent through the artery.

When a stent is expanded in a straight vessel, the links act as connectors and can be excluded from the computational model. Therefore, to minimize the computational costs, the advantage is taken of the 


\begin{tabular}{|c|c|c|c|c|c|}
\hline $\begin{array}{l}\text { Cluster } \\
\text { Number } \\
\end{array}$ & 1 & 2 & 3 & 4 & 5 \\
\hline 1 & 0.058 & 0.079 & 0.110 & 0.141 & 0.161 \\
\hline 0.062 & ST.1 & ST. 6 & ST.11 & ST.16 & ST.21 \\
\hline 0.078 & ST.2 & ST.7 & ST.12 & ST.17 & ST. 22 \\
\hline 0.098 & ST.3 & ST.8 & ST.13 & ST.18 & ST.23 \\
\hline 0.110 & ST.4 & ST.9 & ST.14 & ST.19 & ST.24 \\
\hline 0.133 & ST.5 & ST.10 & ST.15 & ST.20 & ST. 25 \\
\hline
\end{tabular}

TABLE 1. Clustering of selected cross-sectional width, W and thickness, $\mathrm{T}$ (mm).

geometrical repetition of a stent design, and in the present study, only one ring of the stent was selected for the 3D model, Fig. 1b

The analytical model selected for this investigation is composed of a biodegradable magnesium stent ring, a rigid cylinder (representing a non-folded balloon used to expand the stent), an artery and plaque, Fig. 1c. The ring of the stent has twelve arches (six peaks and six valleys) in the circumferential direction, it is $1.5 \mathrm{~mm}$ long and its outer diameter is $2 \mathrm{~mm}$, these parameters are kept unchanged through the optimization process. As for the cross-sectional dimensions of the stent, five strut widths and five strut thicknesses were selected by Latin Hyper Cube Sampling (LHCS) scheme within the bounds stated for these in the literature [15-17] and shown in Eq. 1]

Table 1 shows the parameter values $(\mathrm{W}, \mathrm{T})$ for the 25 stents.

$$
\left\{\begin{array}{l}
0.05 \mathrm{~mm} \leq W \leq 0.17 \mathrm{~mm} \\
0.06 \mathrm{~mm} \leq T \leq 0.14 \mathrm{~mm}
\end{array}\right.
$$

where $W$ and $T$ are strut's width and thickness, respectively. To generate the helical stent ring of specified dimensions and a variable strut width and thickness, a MATLAB user-subroutine code was prepared in order to automatically generate three-dimensional stent geometries.

As for the artery vessel, it is modelled as a straight, hollow cylinder, $2.8 \mathrm{~mm}$ long with an inner diameter of $3.3 \mathrm{~mm}$ and a wall thickness of $0.9 \mathrm{~mm}$. The plaque has a length of $2.2 \mathrm{~mm}$ and its thickness gradually changes from $0.2 \mathrm{~mm}$ at each side to $0.6 \mathrm{~mm}$ at the center [15]. The rigid cylinder within the stent is $2.6 \mathrm{~mm}$ long.

The whole balloon-stent-plaque-vessel FE model used in the present study consisted of a total of 18500 elements. A mesh convergence test was performed to ensure enough mesh refinement. The cylinder has meshed with surface elements and other components (stent, artery and plaque) with solid elements.

To avoid rigid body movements of the system, the ends of the cylindrical balloon and arterial vessel were fixed in axial and circumferential directions. Furthermore, twelve outer nodes on the central cross-section of the stent were constrained longitudinally and circumferentially to prevent motions and yet allowed to freely expand radially.

As for the interaction between the inner stent surface and cylindrical balloon, and the stent outer surface and plaque inner surface, a hard contact formulation for normal behaviour and a frictional coefficient of 0.2 for all tangential behaviour were used, based on De Beule et al. [18. Furthermore, to capture the interaction between the degrading stent and the vessel following the element removal, the special general contact algorithm was incorporated to redefine the faces in contact.

\subsection{MAterials' PROPERTIES AND MODElS}

The stent was assumed to be made of a commercial magnesium alloy AZ31, modelled as a homogeneous, isotropic, elastoplastic material with non-linear isotropic hardening plasticity. The extruded AZ31 has a Poisson's ratio of 0.35 , the yield stress of $138 \mathrm{MPa}$ and ultimate tensile strength (UTS) of $245 \mathrm{MPa}$ at a strain of $17 \%$ 9. The material properties of elements on exposed surfaces of the stent susceptible to corrosion were controlled by damage parameters based on the continuum damage model. In addition to failure due to excessive corrosion, a ductile failure condition was also set for elements in which strains exceed a specified ultimate value. As for the balloon, an isotropic, linear-elastic material model, characterized by a Young's modulus of $920 \mathrm{MPa}$ and a Poisson ratio of 0.4 , was used [19].

A hyper-elastic isotropic constitutive model based on a sixth-order reduced polynomial strain energy density function was used for the vessel tissue and the atherosclerotic plaque. More details can be found in references [8, 9, 20]. Table 2 shows the model parameters for the artery vessel and plaque.

\subsection{Corrosion MOdeL}

In this study, a user prepared explicit solver subroutine for modelling corrosion based on a continuum damage theory was incorporated within a commercial FE code. The continuum damage approach accounts for the effects of corrosion-induced microscale geometric discontinuities by reducing the macroscopic mechanical properties of the material (e.g., stiffness). 


\begin{tabular}{cccccccc}
\hline Constant & $C_{10}(M P a)$ & $C_{20}(M P a)$ & $C_{30}(M P a)$ & $C_{40}(M P a)$ & $C_{50}(M P a)$ & $C_{60}(M P a)$ & $k(M P a)$ \\
\hline Artery & $6.52 \mathrm{E}-3$ & $4.89 \mathrm{E}-2$ & $9.26 \mathrm{E}-3$ & 0.76 & -0.43 & $8.69 \mathrm{E}-2$ & - \\
Plaque & 0.00238 & 0.189 & -0.388 & 3.73 & -2.540 & 0.573 & 4.762 \\
\hline
\end{tabular}

TABLE 2. Constitutive material parameters for strain energy density function of artery and plaque.

The degradation of mechanical properties has been considered through the introduction of a scalar damage parameter, D, as described in Gastaldi et al. [6].

$$
\frac{d D}{d t}=\frac{\delta_{U}}{L_{e}} k_{U}
$$

where, $\delta_{U}$ and $L_{e}$ are the material and finite element characteristic lengths provided by the utility routine for each element and $k_{U}$ represents the corrosion kinetic parameter. In the CDM approach, the effects of geometrical discontinuities can be related to the reduction of macroscopic mechanical properties of the material (e.g., stiffness) through the introduction of damage parameter, $D$, and an effective stress tensor, $\tilde{\sigma}_{i j}$.

The effective stress tensor components $\tilde{\sigma}_{i j}$ can then be defined as:

$$
\tilde{\sigma}_{i j}=\frac{\sigma_{i j}}{1-D}
$$

where $\sigma_{i j}$ is the corresponding undamaged stress tensor component.

Based on the isotropic damage assumption, $D$ is a scalar field that calculates the loss of integrity due to the corrosion for each element. During the corrosion process, the corrosion damage variable $D$ gradually changes from 0 to 1 . In particular, $D=0$ corresponds to an undamaged state and $D=1$ represents a completely damaged or fully corroded state of the material.

In this work, $D$ is only related to the material damage resulting from a localized form of corrosion called pitting corrosion. While the pitting corrosion model can accurately describe the dependency between the fracture time and the applied stress, the uniform corrosion model is not able to predict the fracture time both qualitatively and quantitatively, [9], hence it is not considered in the present study.

The damage evolution law for the case of pitting or heterogeneous corrosion process is $[9]$ :

$$
\frac{d D}{d t}=\frac{\delta_{U}}{L_{e}} \lambda_{e} k_{U}
$$

$\lambda_{e}$ is, for heterogeneous corrosion, the degradation rate of each element, determined for surface elements susceptible to corrosion by a Weibull Distribution Function. In this study, following Grogan et al. [9], $\delta_{U}$ and $k_{U}$ were set equal to $0.017 \mathrm{~mm}$ and $0.026^{h^{-1}}$, respectively.
The corrosion model is implemented in a finite element framework through the development of a user subroutine. To update the state of elements, the user subroutine is called in at each material point in the explicit time integration scheme. The user subroutine determines the element connectivity data and generates a random number through an input file as a pre-processor requirement. The elements on the surface layer, which are susceptible to corrosion, have been determined based on the element connectivity map. At the beginning of each analysis increment, the user subroutine receives stress, strain and time increments from the FE solver and then returns the material properties based on an updated damage parameter at the end of the analysis increment.

When the damage parameter of an element, $D$, reaches 0.9 or the equivalent plastic strain of any element, $\varepsilon_{p l}$, exceeds a strain of $17 \%$ at ultimate tensile strength [9], the element is deleted from the finite element model by this subroutine. After the strongly corroded elements are deleted, the corrosion layer is updated at the end of the time increment so that elements around the removed element are now designated as an updated corrosion layer and exposed to the aggressive environment.

\subsection{VERIFICATION OF CORROSION MODEL}

In this study, the results of the corrosion model simulation, as outlined in section 2.3, were validated against experimental data provided by Grogan et al. 9]. The same foil samples as that of Grogan et al. 9] constructed in the finite element framework were subjected to the pitting corrosion routine, while different uniform tensile stresses, constant throughout the corrosion process, were applied to them, Fig. 2 .

The time required for the foil samples to rupture due to corrosion was then determined and the results are shown in Fig. 3 along with the experimental and FE simulation results of Grogan et al. [9]. The comparison of the results showed a good correlation between the present FE corrosion simulation and experimental results. Therefore, the predicting capability of the corrosion computer code was verified. The $\mathrm{R}^{2}$ values of the result of Grogan, et al. 9 FE simulation and the present research against the experimental result of Grogan, et al. 9] were calculated and the values 0.851 and 0.870 , respectively, were obtained. The obtained values show a satisfying similarity. 


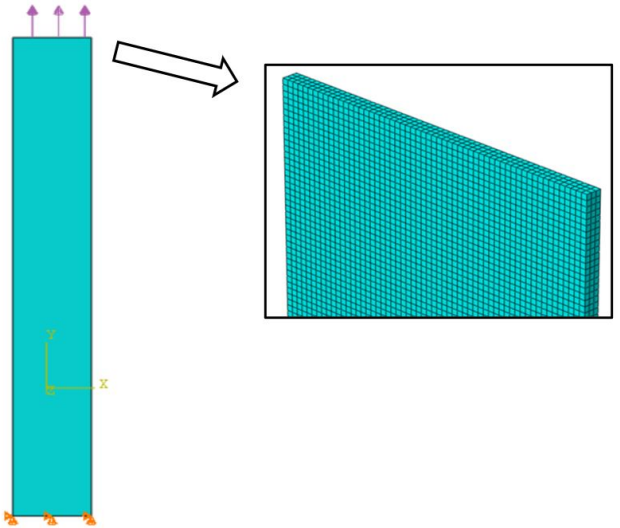

(A).

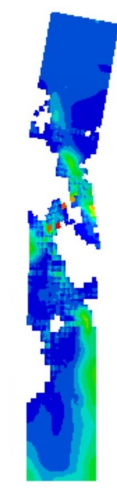

(B).

FiguRE 2. Foil specimen used for verification against Grogan et al. 9] expermental results: (A) FE model, boundary condition and loading; (B) Corroded model.

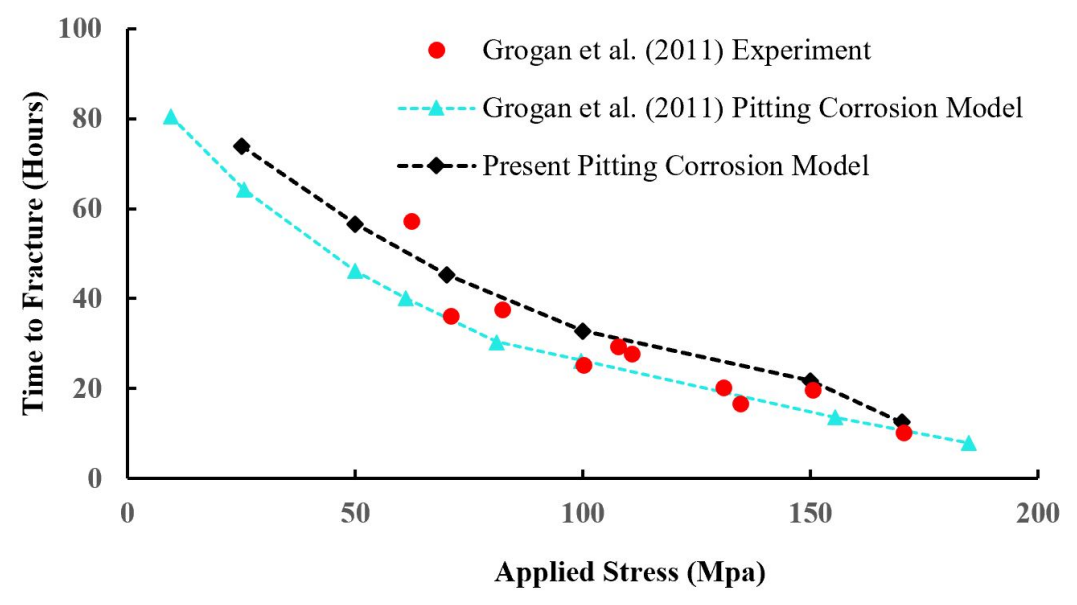

FiguRE 3. Verification of numerical simulation against experimental results.

\section{Results}

\subsection{Stenting Procedure And simulation}

The stenting simulation, consisting of four steps, is shown in Fig. 4. This shows the variation of the outer diameter of stent ST.19, in terms of mm, vs. Normalized Time Unit (NTU). As the first two steps take place in a very short time period, the time scale has been elongated to capture the peculiarities of the first two steps.

In the first step (expansion), after the stent is deployed to the desired point of the artery (that is partially clogged), it is expanded to an outer diameter of $3 \mathrm{~mm}$ by enforcing a radial displacement a driven process on the rigid cylindrical balloon. In the second step (elastic recoil), the cylindrical balloon is contracted elastically in the radial direction. This allows the expanded stent and artery to recoil (and yet, retaining a larger diameter than the original one). In the third step (degradation process), the biodegradable stent undergoes corrosion, however, still acting as a temporary scaffolding for the opened vessel. During this phase, the arterial wall exerts compression on the stent. Finally, in the last step (collapse), the stent collapses due to excessive degradation.

Fig. 5 shows, (a) the stress distribution of the stent after the expansion, (b) at the end of the elastic recoil, and (c) at three time points in the process of the stent degradation; the last state corresponds to the collapsed stent. The four-step stenting procedure depicted in Fig. 4 should be simulated by any realistic numerical algorithm. However, the expansion and elastic recoil steps occur in a very short time as compared to the degradation time, as could be seen in Fig. 4, and therefore, the corrosive attack during these two steps is negligible and thus ignored.

\subsection{SignificAnt PARAMETERS TO BE STUdied}

One of the essential clinical trials' issues regarding the MAS is its high restenosis due to stent's early recoil. Besides this, early and fast degradation of magnesium alloys weakens the radial stiffness of the stent and could be the primary cause for the premature loss of stent scaffolding and recoil. Hence, the radial recoil 


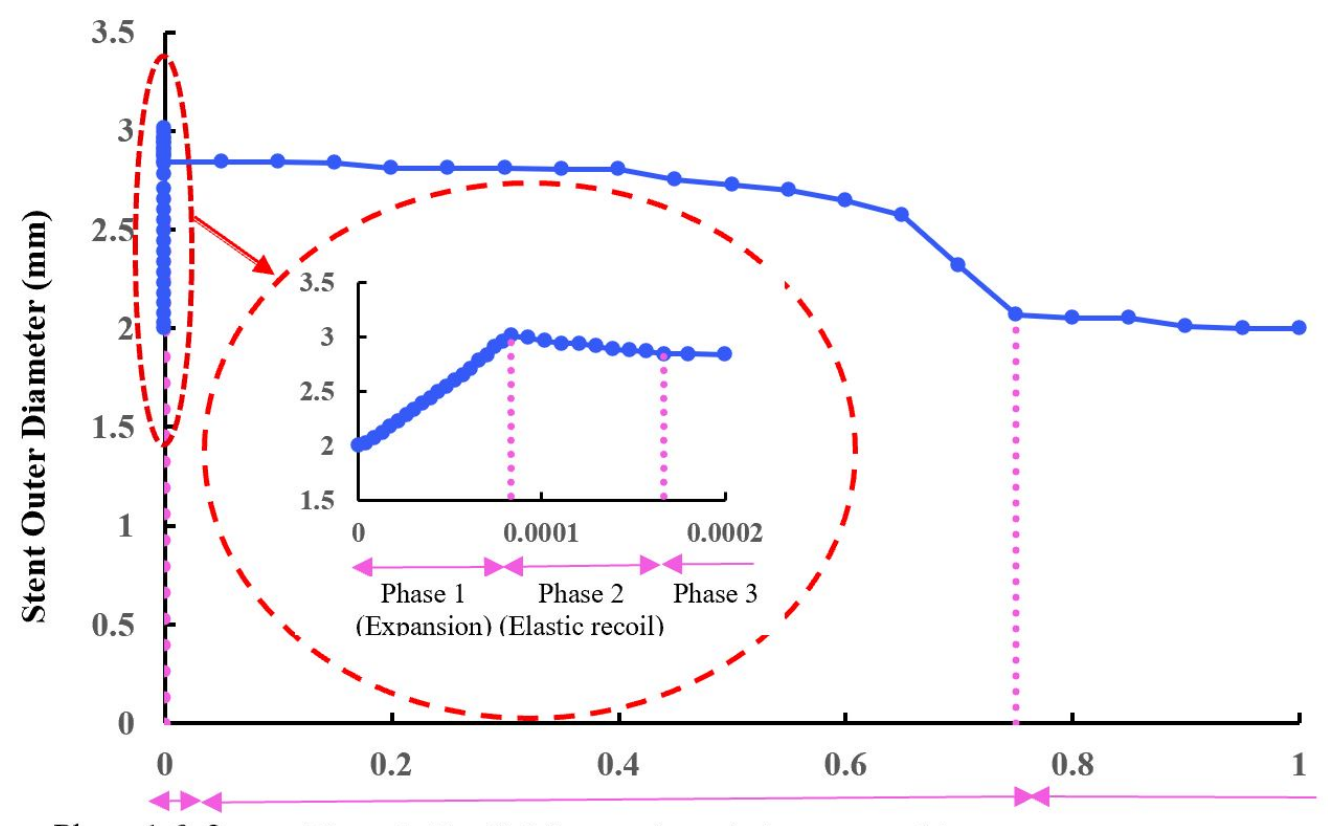

Phase $1 \& 2$ Phase 3 (Scaffolding as degradation proceeds) Phase 4

\section{Normalized Time Unit ( $\left(t^{*}\right)$}

FiguRE 4. Four steps of stenting procedure.
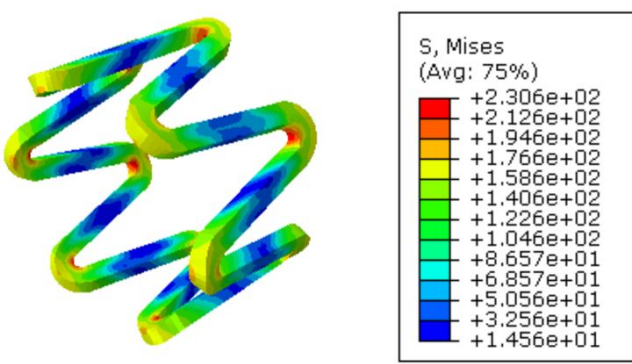

(A).

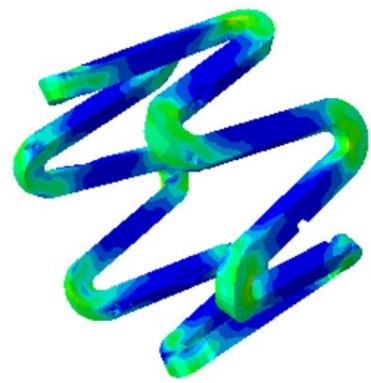

$t^{*}=0.4$

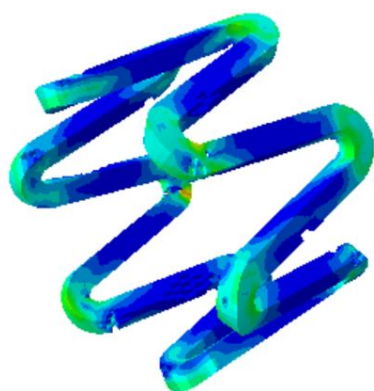

$\mathrm{t}^{*}=0.6$

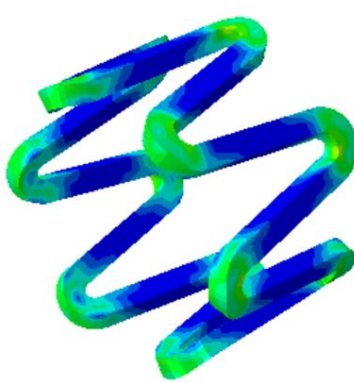

S, Mises

(Avg: 75\%)

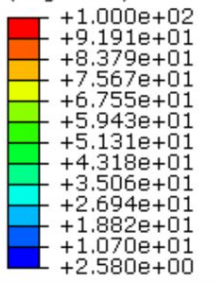

(B).
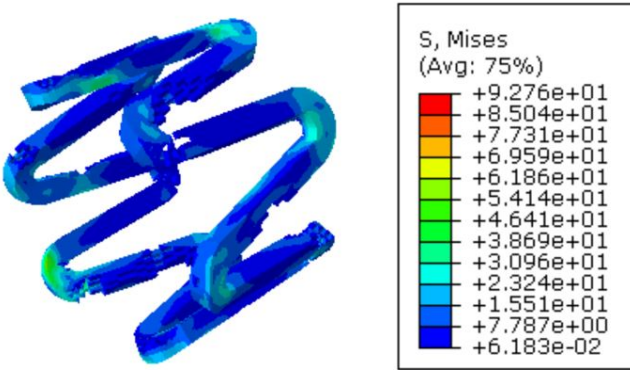

$\mathrm{t}^{*}=0.9$

(C).

Figure 5. Von Mises stress contours at different stages of stenting: (A) At end of expansion stage; (B) At end of recoil stage; (C) At degradation stages corresponding to $t^{*}=0.4,0.6$ and 0.9 . 
and stiffness of the stent are the main mechanical characteristics of biodegradable stents, and any attempt to improve these properties reduces the risk of restenosis [1]. As a result, the recoil and radial stiffness of the stent, as defined in this section, are the key features that would be considered in the present study as a criterion for a subjective optimal design of the stent.

Stent Recoil Percent (SRP) This is defined as the relative difference of the stent diameter at the beginning of the corrosion process (step 2 of section 3.1), $D_{\text {step } 2}^{\text {st }}$, and the stent diameter during the recoil, as the corrosion proceeds (step 3 of section 3.1), $D_{\text {recoil }}^{\text {st }}$, expressed in percentage, see Eq. 5 .

$$
S R P(t)=\frac{D_{\text {step } 2}^{\text {st }}-D_{\text {recoil }}^{\text {st }}(t)}{D_{\text {step } 2}^{\text {st } 2}} \times 100 \text { in } \%
$$

Stent Radial Stiffness (SRS) This is defined as the ratio of the average contact normal force exerted on the outer surface of the stent by the plaque, $\mathrm{F}_{\mathrm{c}}$, to the external radius of the stent, $\mathrm{R}$, as the corrosion proceeds, see Eq. 6.

$$
S R S(t)=\frac{F_{c}(t)}{R(t)} \quad \text { in } N / m m
$$

Mass Loss (ML) In addition to the two previously defined parameters, a third one, mass loss, is considered in the optimum design. The mass loss percent of the whole stent model at any time is calculated based on the damage parameter $\mathrm{D}$ value averaged over all of the elements times 100, see Eq. 7

$$
\begin{aligned}
& M L(t)= \\
& =\left(\frac{1}{N_{\text {elements }}}\right) \times\left(\sum_{i=1}^{N_{\text {elements }}} D_{i}(t)\right) \times 100 \quad \text { in } \%
\end{aligned}
$$

\subsection{Stent APPLiCATION}

In this study, the 25 stents of different cross-sectional dimensions, as categorized in five clusters of fixed width and variable thicknesses (Table 1), were modelled in the prepared computer environment. Fig. 6 shows the variation of SRP (in percentage) with a normalized time unit, NTU, $\mathrm{t}^{*}$ for clusters 1 to 5 , respectively. It is to be noted that the NTU of Fig. 6 is measured from the end of phase 2 of Fig. 4

Inter-Vascular Ultra-Sound (IVUS) imaging of the absorbable magnesium stent (AMS) in human coronaries has shown that recoil is a significant contributor to restenosis. This imaging has also shown that the average value of recoil at the time of a complete stent degradation is about $27 \%$ [21, 22]. Because of this, a horizontal line corresponding to the SRP of $27 \%$ is drawn on Fig. 6. However, the stents lose their scaffolding ability approximately at a normalized time unit, $\mathrm{t}^{*}$, of 0.95 , beyond which the stent degraded completely. Therefore, a vertical line corresponding to $t^{*}$ of 0.95 is also drawn on this figure.

\section{Discussion}

In this research, 25 stents having different crosssectional dimensions were subjected to heterogeneous corrosion and their performance with regard to radial recoil, stiffness, and mass loss were monitored. The two-tier process of determining the optimum stent design by using these simulation results is described. In this regard, any stent showing SRP greater than $27 \%$ prior to $t^{*}$ of 0.95 was excluded from the pool of admissible stents. In reference to Fig. 6a, 6b and $6 \mathrm{c}$, it is concluded that all stents of clusters 1 to 3 have to be removed from the pool. But stents ST.19 and ST.20 of cluster 4, Fig. 6d, showed SRP lower than $27 \%$ at $\mathrm{t}^{*}$ of 0.95 . These are two of the eligible candidate stents. From Fig. 6e it is concluded that stents ST.24 and ST.25 of cluster 5 also show the same feature.

The recoil percent history of the four stents, ST.19, ST.20, ST.24 and ST.25 are shown in Fig. 7a These cross the vertical line corresponding to $\mathrm{t}^{*}$ of 0.95 at $22.555,23.736,24.808$, and 24.868, respectively, showing that all the stents retain an adequate diameter at the time of the stent collapse due to degradation. From $t^{*}=0$ to approximately 0.75 , the four stents show almost the same recoil behaviour, but from $t^{*} \approx$ 0.75 onward, ST.19 shows a lesser recoil percentage. No definite conclusion with regard to the optimum stent cross-section can be drawn just by considering the SRP.

As stated in section 3.2 , radial stiffness is another important characteristic of MAS, which directly controls the scaffolding ability of the stent. Nevertheless, no criterion or specified values regarding the radial stiffness has been reported in the literature. As both recoil and radial stiffness are essential properties of the stent if it has to function properly, the variation with a normalized time unit of radial stiffness of just those stents complying with recoil requirement, i. e., ST.19, ST.20, ST.24, and ST.25 are shown in Fig. 7b In this figure, the vertical line corresponding to $t^{*}$ of 0.95 is drawn; this is the time the stent losses its scaffolding ability. To make drawing conclusions easy, the SRP and SRS values of the four stents listed at $\mathrm{t}^{*}$ of 0.95 are tabulated in Table 3 along with the mass loss percent. Column 2 of this table shows that ST.19 has the lowest recoil, while column 4 shows that ST.24 has the largest stiffness.

In column 3, the recoil ratio relative to ST. 19 (which has the smallest recoil percentage) is shown, in columns 5 and 6 , radial stiffness ratios relative to ST.19 and ST. 24, respectively, are shown. The values in columns 3 and 6 indicate that ST.24 has an almost $10 \%$ higher recoil as compared to ST.19, while ST.19 has an almost $34 \%$ lower stiffness as compared to 


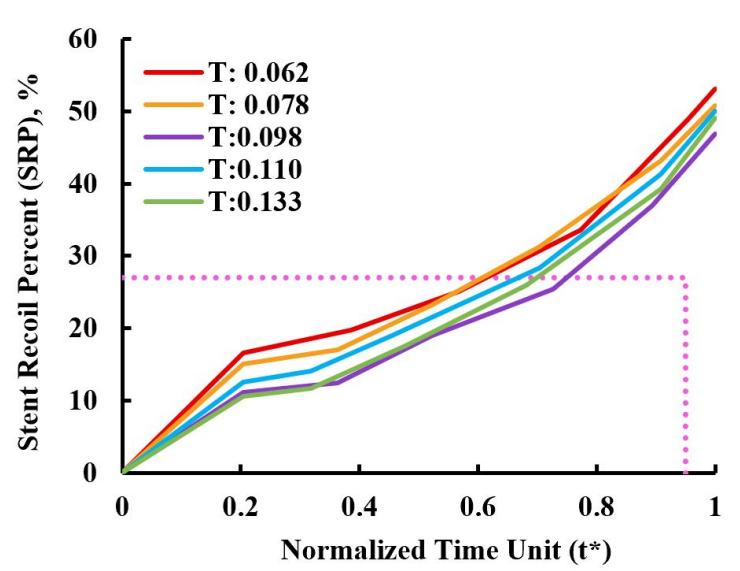

W: 0.058

(A).

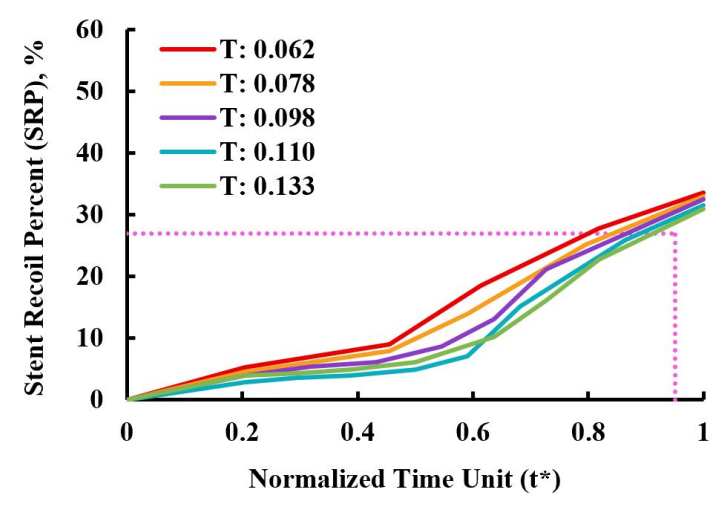

W: 0.110

(c).

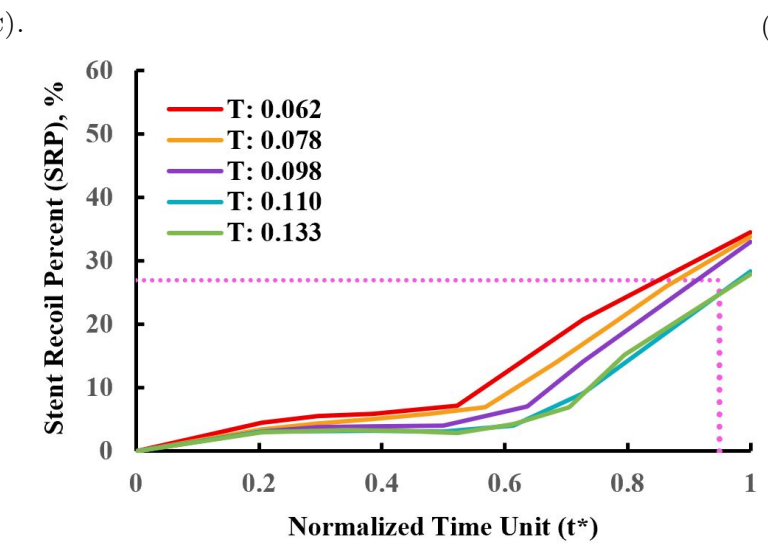

W: 0.161

(E).

Figure 6. Recoil of stents of five clusters as degradation proceeds: (A) Cluster 1; (B) Cluster 2; (C) Cluster 3; (D) Cluster 4; (E) Cluster 5 (all W's and T's are in $\mathrm{mm}$ ).

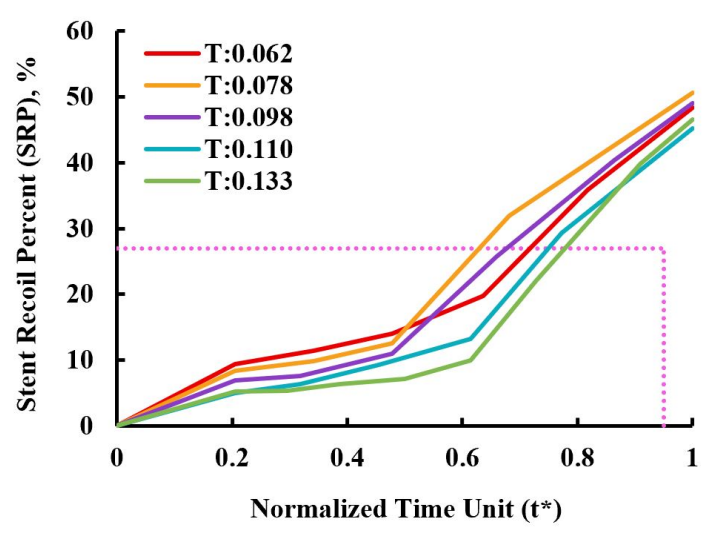

W: 0.079

(B).

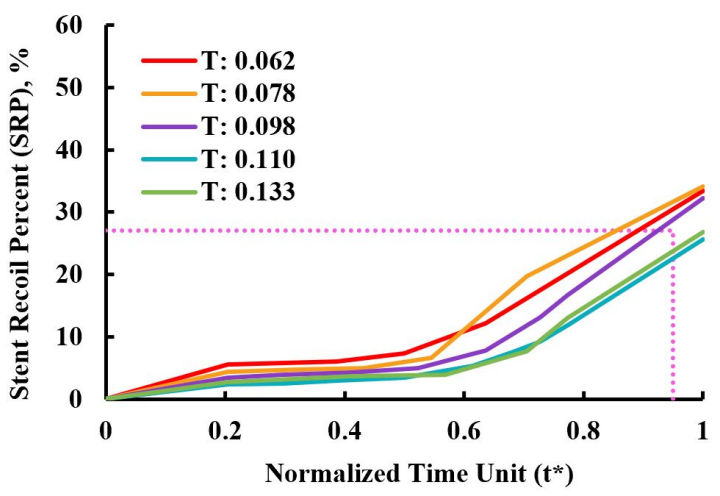

W: 0.141

(D). 


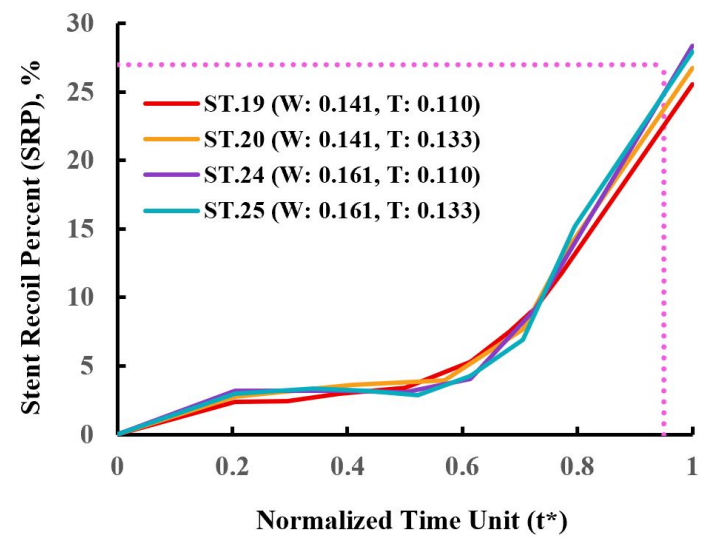

(A).

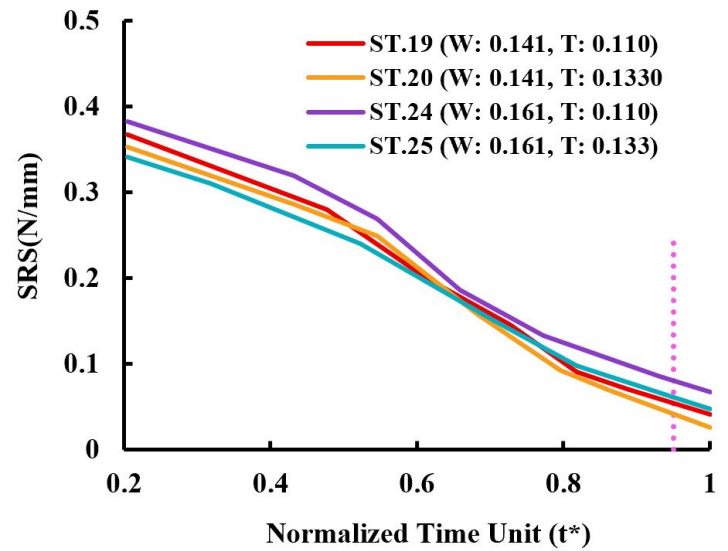

(в).

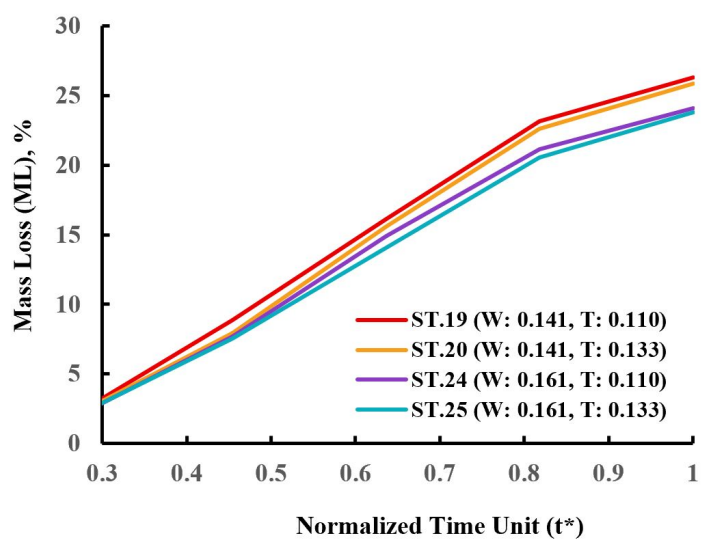

(C).

Figure 7. Variation of SRP, SRS and ML of stents ST.19, ST.20, ST.24, and ST.25 with time: (A) Variation of SRP; (B) Variation of SRS; (C) Variation of ML (all W's and T's are in mm).

ST.24. This shows that the optimum stent design would be ST.24, even though it requires almost $14 \%$ more material used for its production as compared to ST.19.

Besides the recoil and stiffness of the stent, the variation of percentage mass loss of stents due to degradation with time (as defined in section 3.2 was monitored and the results for ST.19, ST.20, ST.24, and ST.25 are shown in Fig. 7c The percentage loss corresponding to the NTU of 0.95 for these four stents is listed in column 7 of Table 3 . The $8.5 \%$ smaller mass loss percentage of ST.24 as compared to ST.19 is another indication of the superiority of ST.24 over the ST.19.

Erbel et al. 1] have shown, through a clinical trial of coronary implantations of absorbable magnesium stents, that the mean and standard deviation of instent diameter stenosis percentage would be 48.37 and 17.00, respectively. Considering these numbers, it can be concluded that the $27 \%$ recoil percentage reported by Waksman [21] and Waksman et al. [22] and used by the authors to this point for finding the optimum profile for stents, ensures an almost $90 \%$ proper functionality during the required scaffolding period. To study the effect of a higher probability of stenosis, say $15.87 \%$, a recoil percent of $48.37-17=$ $31.37 \%$ is used instead of $27 \%$ in screening the stents. The result is shown in Fig. 8a, with Stent Recoil Percent (SRP) as ordinate and Normalized Time Unit (NTU) as abscissa.

Five more stents than the four previously complying with the criterion of $27 \%$ recoil percentage are included in the batch; overall, nine stents have a lower recoil percentage than 31.37 at $\mathrm{t}^{*}$ of 0.95 . To find the optimum profile design, the variation of the stent radial stiffness (SRS) versus the time is shown in Fig. $8 \mathrm{~b}$ for selected six of the nine stents passing the recoil criterion. It is clearly evident that ST.24 has a higher stiffness than others, and therefore, would be the optimum choice. This indicates that the selection of the recoil percentage does not alter the optimum design and ST.24 with a $0.110 \mathrm{~mm}$ thickness and a $0.161 \mathrm{~mm}$ width would be the optimum choice with an almost $90 \%$ probability of surpassing the recoil criteria and having an adequate stiffness, while not having the largest cross-section.

\section{Conclusion}

In this study, recoil and radial stiffness of stents were considered as the criteria for a subjective optimal 


\begin{tabular}{ccccccc}
\hline ST \# & $\begin{array}{c}\text { SRP at t* }=0.95 \\
(\%)\end{array}$ & Recoil Ratio & $\begin{array}{c}\text { SRS at t* } \mathrm{t}^{*}=0.95 \\
(\mathrm{~N} / \mathrm{mm})\end{array}$ & Radial Stiffness Ratio & $\begin{array}{c}\text { Mass Loss (ML) } \\
(\%)\end{array}$ \\
\hline 1 & 2 & 3 & 4 & 5 & 6 & 7 \\
\hline ST.19 & 22.555 & 1 & 0.053 & 1 & 0.6625 & 25.438 \\
ST.20 & 23.736 & 1.052 & 0.041 & 0.771 & 0.5125 & 24.986 \\
ST.24 & 24.808 & 1.099 & 0.080 & 1.485 & 1 & 23.281 \\
ST.25 & 24.868 & 1.102 & 0.061 & 1.134 & 0.7625 & 22.912 \\
\hline
\end{tabular}

TABLE 3. Comparison of recoil percent and radial stiffness of ST.19, ST.20, ST.24 and ST.25.

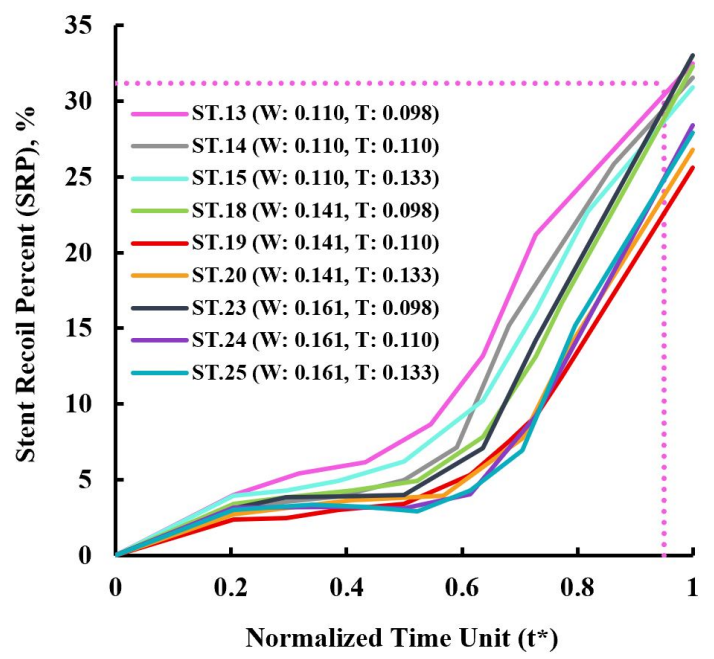

(A).

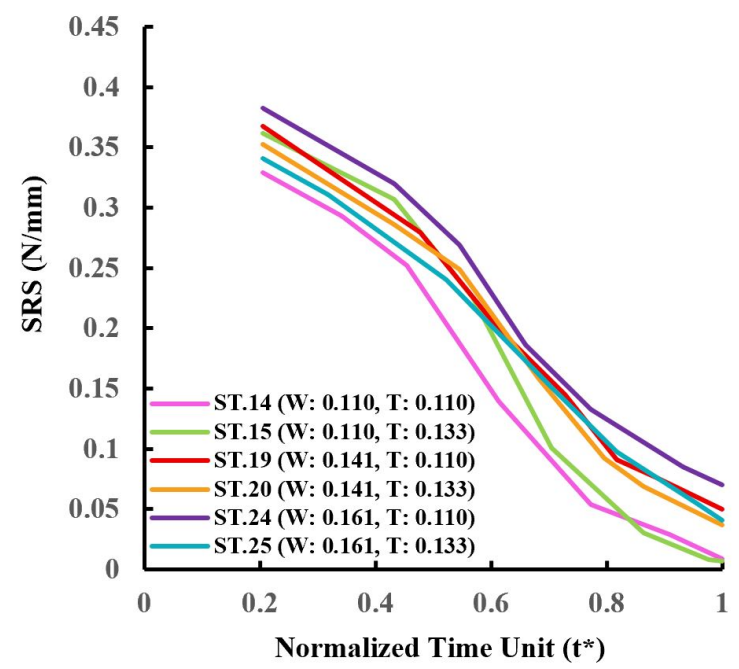

(в).

FigURE 8. Variation of SRP and SRS: (A) Variation of SRP of stents having lesser SRP than 31.37\%; (B) Variation of SRS of stents ST.14, ST.15, ST.19, ST.20, ST.24, and ST.25 (all W's and T's are in mm) .

design of stents. The results of the analysis of 25 stents showed that the optimum choice, ST.24, with a $0.110 \mathrm{~mm}$ thickness and a $0.161 \mathrm{~mm}$ width, had at least $24 \%$ higher stiffness than others and passed the recoil criteria with an almost $90 \%$ probability of proper functionality. Although ST.24 requires an almost $14 \%$ more material used for its production as compared to ST.19, it has $8.5 \%$ and $6.8 \%$ smaller mass loss percent as compared to ST.19 and ST.20, respectively, and thus it would be the final choice even though it does not have the largest cross-section within the cluster set selected by the LHCS. It's noteworthy that the optimum choice has a thinner cross-sectional profile than the commercially available magnesium alloy stents and this makes the stent delivery much easier and lowers the risk of restenosis as compared to commercially available thicker MAS stents.

To the best of authors' knowledge, this is the first attempt for subjectively determining (without using optimization codes and extensive computational effort) the optimal cross-section of a biodegradable magnesium alloy stent undergoing heterogeneous corrosion. It should also be noted that the method set forth in the present research and applied to sine-wave magnesium alloy (AZ31) stent could, as well, be applied to any stent with a different configuration and mate- rial provided its mechanical properties and corrosion parameters are known.

\section{REFERENCES}

[1] R. Erbel, C. Di Mario, J. Bartunek, et al. Temporary scaffolding of coronary arteries with bioabsorbable magnesium stents: a prospective, non-randomised multicentre trial. The Lancet 369(9576):1869-1875, 2007. https://doi.org/10.1016/s0145-4145(08) 04009-4.

[2] M. Haude, R. Erbel, P. Erne, et al. Safety and performance of the drug-eluting absorbable metal scaffold (DREAMS) in patients with de-novo coronary lesions: 12 month results of the prospective, multicentre, first-in-man BIOSOLVE-I trial. The Lancet

381(9869):836-844, 2013. https://doi.org/10.1016/s0140-6736(12)61765-6

[3] B. D. Gogas. Bioresorbable scaffolds for percutaneous coronary interventions. Global Cardiology Science and Practice 2014(4), 2015. https://doi.org/10.5339/gcsp.2014.55

[4] P. E. McHugh, J. A. Grogan, C. Conway, E. Boland. Computational modeling for analysis and design of metallic biodegradable stents. Journal of Medical Devices 9(3):030946, 2015. https://doi.org/10.1115/1.4030576

[5] T. Hu, C. Yang, S. Lin, et al. Biodegradable stents for coronary artery disease treatment: Recent advances and 
future perspectives. Materials Science and Engineering: C 91:163-178, 2018. https://doi.org/10.1016/j.msec.2018.04.100

[6] D. Gastaldi, V. Sassi, L. Petrini, et al. Continuum damage model for bioresorbable magnesium alloy devices - application to coronary stents. Journal of the Mechanical Behavior of Biomedical Materials 4(3):352-365, 2011. https://doi.org/10.1016/j.jmbbm.2010.11.003

[7] W. Wu, S. Chen, D. Gastaldi, et al. Experimental data confirm numerical modeling of the degradation process of magnesium alloys stents. Acta Biomaterialia 9(10):8730-8739, 2013. https://doi.org/10.1016/j.actbio.2012.10.035.

[8] W. Wu, D. Gastaldi, K. Yang, et al. Finite element analyses for design evaluation of biodegradable magnesium alloy stents in arterial vessels. Materials Science and Engineering: B 176(20):1733-1740, 2011. https://doi.org/10.1016/j.mseb.2011.03.013

[9] J. A. Grogan, B. J. O'Brien, S. B. Leen, et al. A corrosion model for bioabsorbable metallic stents. Acta Biomaterialia 7(9):3523-3533, 2011. https://doi.org/10.1016/j.actbio.2011.05.032.

[10] W. Wu, L. Petrini, D. Gastaldi, et al. Finite element shape optimization for biodegradable magnesium alloy stents. Annals of Biomedical Engineering 38(9):2829-2840, 2010. https://doi.org/10.1007/s10439-010-0057-8.

[11] J. A. Grogan, S. B. Leen, P. E. McHugh. Optimizing the design of a bioabsorbable metal stent using computer simulation methods. Biomaterials 34(33):8049-8060, 2013. https:

//doi.org/10.1016/j.biomaterials.2013.07.010

[12] C. Chen, J. Chen, W. Wu, et al. In vivo and in vitro evaluation of a biodegradable magnesium vascular stent designed by shape optimization strategy. Biomaterials 221:119414, 2019. https: //doi.org/10.1016/j.biomaterials.2019.119414

[13] X. Ni, Y. Zhang, C. Pan. The degradable performance of bile-duct stent based on a continuum damage model: A finite element analysis. International Journal for Numerical Methods in Biomedical Engineering p. 36:e3370, 2020. https://doi.org/10.1002/cnm.3370
[14] L. Petrini, F. Migliavacca, F. Auricchio, et al. Numerical investigation of the intravascular coronary stent flexibility. Journal of Biomechanics 37(4):495-501, 2004. https://doi.org/10.1016/j.jbiomech.2003.09.002

[15] S. Pant, N. W. Bressloff, G. Limbert. Geometry parameterization and multidisciplinary constrained optimization of coronary stents. Biomechanics and Modeling in Mechanobiology 11(1-2):61-82, 2012. https://doi.org/10.1007/s10237-011-0293-3.

[16] H. Li, J. Gu, M. Wang, et al. Multi-objective optimization of coronary stent using Kriging surrogate model. BioMedical Engineering OnLine 15:148, 2016. https://doi.org/10.1186/s12938-016-0268-9.

[17] C. McCormick. Overview of cardiovascular stent designs. In Functionalised Cardiovascular Stents, pp. 3-26. Woodhead Publishing, 2018. https: //doi.org/10.1016/b978-0-08-100496-8.00001-9

[18] M. De Beule, S. Van Cauter, P. Mortier, et al. Virtual optimization of self-expandable braided wire stents. Medical Engineering \& Physics 31(4):448-453, 2009. https://doi.org/10.1016/j.medengphy.2008.11.008

[19] M. De Beule, P. Mortier, S. G. Carlier, et al. Realistic finite element-based stent design: the impact of balloon folding. Journal of Biomechanics 41(2):383-389, 2008. https://doi.org/10.1016/j.jbiomech.2007.08.014

[20] F. Gervaso, C. Capelli, L. Petrini, et al. On the effects of different strategies in modelling balloonexpandable stenting by means of finite element method. Journal of Biomechanics 41(6):1206-1212, 2008. https://doi.org/10.1016/j.jbiomech.2008.01.027

[21] R. Waksman. Current state of the absorbable metallic (magnesium) stent. Euro Intervention: Journal of EuroPCR in Collaboration with the Working Group on Interventional Cardiology of the European Society of Cardiology 5:F94-F97, 2009. https://doi.org/10.4244/eijv5ifa16.

[22] R. Waksman, R. Erbel, C. Di Mario, et al. Early-and long-term intravascular ultrasound and angiographic findings after bioabsorbable magnesium stent implantation in human coronary arteries. JACC: Cardiovascular Interventions 2(4):312-320, 2009. https://doi.org/10.1016/j.jcin.2008.09.015 